\title{
Una revisión de los estilos de vida de estudiantes universitarios
}

\author{
MSc. Marta Lorena Espinoza Lara \\ Recinto Universitario Rubén Darío \\ UNAN-Managua \\ mespinozaatram@gmail.com
}

PhD. Jairo Vanegas López

Recinto Universitario Rubén Darío

UNAN-Managua

jvanegas07@gmail.com

DOI: http://dx.doi.org/10.5377/torreon.v6i16.6554

Palabras clave: estilo de vida, estudiante, determinantes sociales, universidad

\section{RESUMEN}

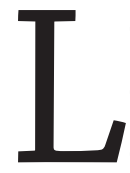

os estilos de vida son los hábitos y conductas que modulan nuestra actividad cotidiana y rigen nuestra forma de afrontar los requerimientos, exigencias y retos que se nos presentan durante las diferentes etapas de nuestra vida. Objetivo: Indagar sobre los estilos de Vida y los estilos de vida de estudiantes universitarios según la evidencia disponible. Metodología: Se realizó una revisión bibliográfica, electrónica de artículos vinculados a estilos de vida en población universitaria a partir del año 2004. Adicionalmente, se recabó información referida al concepto de estilos de vida y se recopilaron antecedentes estadísticos disponibles de investigaciones realizadas en Nicaragua. Resultados: La mayor parte de los trabajos desarrollados han estudiado, de manera descriptiva, los estilos de vida. La alimentación, el consumo de tabaco, alcohol y drogas, son las prácticas más estudiadas. Conclusión: Los jóvenes universitarios son una población vulnerable que debe ser investigada de manera particular, para crear y evaluar programas de intervención acorde a sus necesidades. 


\section{INTRODUCCIÓN}

En las últimas décadas del siglo XX, el mundo experimentó grandes avances científicos y tecnológicos en el campo de la biomedicina que han tenido gran repercusión en la mejora de la salud de la Humanidad. Sin embargo, al inicio del siglo XXI, no podemos mostrarnos tan optimistas y resulta sumamente preocupante constatar que, existen muchos problemas de salud no resueltos y que las desigualdades en este ámbito se están acentuando de forma alarmante, entre el mundo desarrollado y los países de renta más baja (Fundación BBVA, 2007).

Las enfermedades no transmisibles (ENT) representan uno de los mayores desafíos del siglo XXI para la salud y el desarrollo, tanto por el sufrimiento humano que provocan como por las consecuencias sociales y económicas que ocasionan en todos los países, pero son particularmente devastadoras en las poblaciones pobres y vulnerables. Cada día, personas más jóvenes están siendo afectadas por estas enfermedades que comparten factores de riesgo comunes como es el tabaquismo, la inactividad física, el sobrepeso, la obesidad, el uso nocivo del alcohol y la dieta no saludable (Organización Mundial de la Salud, 2014).

En el año 2012 a nivel mundial, las enfermedades no transmisibles ocasionaron 38 millones de muertes, de las cuales, más del $40 \%$ de ellas (16 millones) fueron muertes prematuras ocurridas antes de los 70 años de edad (Organización Mundial de la Salud, 2014).

En Nicaragua, por su parte, las ENT son la causa del $73 \%$ de las muertes y la probabilidad de morir entre los 30 y 70 años debido a las ENT principales es del 19 \% (Organización Mundial de la Salud, 2014).

De igual manera, la Encuesta de Diabetes, Hipertensión y Factores de riesgo de Enfermedades Crónicas, Managua 2010, muestran datos de riesgo significativo a la salud. Los resultados indican que el 25,2 \% de los entrevistados presentaron hipertensión. Se encontró sobrepeso (IMC >= 25) en el $65 \%$ del total de los encuestados (37\% sobrepeso y $28 \%$ obesidad), siendo las mujeres más afectadas que los hombres (71 \% y 59 \% respectivamente). El 33 \% realiza actividades físicas baja (Pan American Health organization, 2010).

Otros factores de riesgo estudiados fueron, el tabaquismo y el uso de alcohol. Un $21 \%$ de los encuestados aceptó ser fumadores y un 72 \% admitió haber consumido cinco o más bebidas alcohólicas en las cuatro semanas previas a la encuesta, con predominio de los hombres de 77.5\% versus $60.7 \%$ entre las mujeres. Se evidenció una alta prevalencia de tabaquismo y alcoholismo en menores de 40 años y ambos factores de prevalencia es mayor en el sexo masculino que en el femenino (Pan American Health organization, 2010). 
Un 25 \% de los encuestados reportó agregar sal adicional a los alimentos ya preparados. Un 62 \% de los encuestados reportó el uso de aceite como la forma más frecuente de preparar los alimentos; este factor fue reportado mayormente por personas del grupo de 20 a 39 años.

Datos de la OMS-ENT perfiles de países, 2014, reporta que el MINSA no cuenta con un sistema nacional de respuesta a las ENT.

Los antecedentes expuestos anteriormente cobran importancia en la población universitaria, tanto es así, que los estudiantes no solo están inmersos en los cambios de la tecnología, de la información y en la creación del conocimiento, sino en cambios sociales como adicciones, violencia social, enfermedades no transmisibles y conductas alimentarias de riesgo, debido a la escasa actividad física y mala calidad en la dieta, influenciados por el desempeño, la exigencia académica y los horarios de clase discontinuos, en una relación de causalidad que puede llevar a la intensificación de riesgos para la salud.

Es importante mencionar que, las ENT se pueden prevenir y controlar a través de cambios en los estilos de vida, las políticas públicas e intervenciones de salud. El control o influencia depende esencialmente de nosotros mismos, de nuestra fuerza de voluntad y compromiso personal, depende de fomentar patrones de conducta basados en promover determinados hábitos saludables, eliminar ciertas costumbres nocivas, configurando un estilo de vida que, positiva o negativamente, pueden condicionar nuestra salud.

Dado entonces que, los estilos de vida de una persona están determinados por las condiciones en las que vive, es muy importante indagar sobre los estilos de vida de los diferentes grupos sociales que convergen dentro de la universidad. El objetivo de este estudio es indagar sobre los estilos de Vida y los estilos de vida de estudiantes universitarios, según la evidencia disponible.

\section{MATERIAL Y MÉTODO}

La revisión fue efectuada mediante búsqueda electrónica de todos aquellos artículos sobre los estilos de vida relacionados con la salud de estudiantes universitarios hispanoamericanos. La base de datos utilizada para la búsqueda fue LILACS en SCIELO, HINARI e ISI. Los descriptores de búsqueda fueron estilos de vida, estudiantes, determinantes sociales, universidad.

La selección de los artículos originales se efectuó considerando un periodo de al menos 10 años publicados, durante el periodo 2004-2014. Se analizaron los resultados y los antecedentes se incorporaron en una base de datos a fin de encontrar similitudes y diferencias para la organización del presente artículo. 


\section{RESULTADOS Y DISCUSIÓN}

A pesar de la creciente importancia sobre el efecto de los estilos de vida en el mundo moderno, el concepto de estilo de vida no constituye un ámbito de estudio nuevo. En este sentido, es importante denotar la contribución que han hecho las ciencias sociales y la medicina social, en procurar esclarecer el concepto de estilos de vida, sus aplicaciones en diversos campos como la salud, específicamente en el ámbito de sus políticas, la epidemiología, la medicina preventiva y la educación en salud, entre otros.

Según revisiones efectuadas, como las realizadas por Ansbacher (1976), Abel (1991) o Coreil et al (1992), el concepto se remonta a finales del siglo XIX, cuando Karl Marx y Max Weber ofrecieron una visión sociológica del estilo de vida, enfatizando en que las variables sociales eran los determinantes de la adopción y mantenimiento de un estilo de vida determinado. Desde la orientación sociológica, la mayoría de las definiciones convergen al entender los estilos de vida como un patrón de actividades o conductas que los individuos eligen adoptar entre aquellas que están disponibles en función de su contexto social (Ramos, 2011).

Durante la segunda mitad del siglo XX, el término "estilos de vida" tuvo mayor impacto en el área de salud. Las investigaciones realizadas adoptan una perspectiva médico epidemiológica. Desde este paradigma, la comunidad médica defendía que las personas practicaban estilos de vida insanos por su propia voluntad. Es decir, el modelo no reconocía la importancia del contexto social y de los factores psicológicos como condicionantes de la adquisición de los estilos de vida.

Los estilos de vida individuales, dicen Laguna, García Salamanca y Tapiero Paipa (2012), están caracterizados por patrones de comportamiento identificables, pueden ejercer un efecto profundo en la salud de un individuo y en la de otros. Si la salud ha de mejorarse permitiendo a los individuos cambiar sus estilos de vida, la acción debe ir dirigida, no solamente al individuo, sino también a las condiciones sociales de vida que interactúan para producir y mantener esos patrones de comportamiento. Sin embargo, es importante reconocer que no existe un estilo de vida "óptimo" al que puedan adscribirse todas las personas. La cultura, los ingresos, la estructura familiar, la edad, la capacidad física, el entorno doméstico y laboral, harán más atractivas, factibles y adecuadas determinadas formas y condiciones de vida. Por ello, la estrategia de crear ambientes favorables para la salud se centra en gran medida en la necesidad de mejorar y cambiar las condiciones de vida para apoyar la salud.

En este sentido, la OMS, en la trigésimo primera sesión de su Comité Regional para Europa, definió "estilo de vida" como una forma de vida que se basa en patrones de comportamientos identificables, determinados por la interacción entre las características personales individuales, las interacciones sociales y las condiciones de vida socioeconómicas y ambientales. Además, se comentó la necesidad de diferenciar entre estilo de vida y estilo de vida saludable y se enfatizó 
sobre la importancia de abordar el estudio del estilo de vida saludable desde un enfoque más social que médico (Veny M. B., 2000).

\section{Estilos de vida en la población universitaria}

En la investigación Salazar y Arrivillaga Quintero (2004) de la universidad de Bogotá, Colombia, encontró con relación al consumo de alcohol, tabaco y otras drogas, que los jóvenes tienen estilos de vida poco saludables y que no existe diferencia significativa entre quienes tienen prácticas saludables y quienes no la tienen. Estos resultados conducen a la discusión sobre los focos y prioridades de intervención con jóvenes en una institución universitaria.

Castaño Castrillón (2010), de la facultad de Psicología de la Universidad de Manizales, Colombia, exploró algunos componentes de los estilos de vida de los estudiantes y encontró que la edad promedio fue de 21,42 años, $85,4 \%$ son solteros y 82 \% pertenecen al género femenino; del $64,7 \%$ se identificó un $65,1 \%$ de no fumadores; $26,9 \%$ no consumen alcohol; 34,6\% presenta problemas con el alcohol y un 21,1\% presenta dependencia del alcohol. Si bien, el $65 \%$ manifiesta protegerse contra ETS y el 87,2 \% se protege contra embarazo, esta protección no se da siempre.

Grimaldo (2010), de la universidad San Martín de Porres de Lima, Perú, determinó la relación entre los dominios de bienestar económico, amigos, vecindario y comunidad pareja, vida familiar, ocio, medios de comunicación, religión y calidad de vida y tres factores del estilo de vida saludable (actividad deportiva, consumo de alimentos y sueño y reposo), no se encontró correlación entre calidad de vida y actividad deportiva. Se halló correlación entre calidad de vida del dominio salud y consumo de alimentos en el grupo de edad más joven. Se observó correlación moderada entre algunos dominios de la calidad de vida (amigos, vida familiar, ocio y religión).

Bacerra Bulla (2011), de la Carrera de Medicina de la Universidad Nacional de Colombia, determinó algunos estilos de vida de estudiantes de pregrado admitidos al primer semestre de la Carrera, en la que encuentran que, aproximadamente la mitad de los estudiantes dormía entre cuatro y seis horas y el restante lo hacía por más tiempo; más de la mitad de los estudiantes que trabajaba dormía de cuatro a seis horas diarias, mientras que cerca de la mitad de los que no trabajaba dormía siete o más horas. La mayoría de los estudiantes manifestó no presentar estrés antes de su ingreso a la universidad. Los estudiantes que afirmaron sentir algún nivel de estrés lo catalogaron como bajo o medio. El 61,1 \% de los estudiantes no consumía alcohol. El 42,2 \% de los hombres entre 14-18 años consumía alcohol, comparado con el 15,6\% de las mujeres de esta edad. El 89 \% de los estudiantes no fumaba y el $44 \%$ que fumaba, lo hacía de manera ocasional.

También García Salamanca, Tapiero Paipa y Ramos C., (2012) encuentran que, los jóvenes universitarios se encuentran expuestos a una serie de factores que los predispone a adoptar conductas nocivas para la salud y aumentar el riesgo de padecer enfermedades crónicas no transmisibles. Por esto, es necesario crear consciencia e implementar estrategias que promuevan 
el cambio hacia los estilos de vida saludables, permitiendo mitigar efectos e impactando en la calidad de vida de cada uno de los individuos.

Hernández Pozo, Jiménez Martínez y Durán Díaz (2014) buscaron analizar la relación entre estilo de vida (EV) y el síndrome metabólico (SM) en estudiantes universitarios, así como establecer si existen diferencias por sexo, encontrando SM en 4,63 \% y obesidad en $36.65 \%$, con mayor frecuencia en las mujeres. El EV predominante fue bueno y mejor en hombres que en mujeres. La relación entre género, obesidad y SM fue significativa para la población estudiada.

Asimismo, Laguando y Gómez Díaz (2014) determinaron los estilos de vida en estudiantes de enfermería de la universidad de Colombia, encontrando que el género predominante fue el femenino, la edad promedio correspondió a 16 mínima y máxima de 38 años, concluyéndose que, en la etapa universitaria, diferentes factores interfieren para mantener una vida saludable, los datos encontrados potencian las conductas de riesgo para enfermedades crónicas no transmisibles, que pueden interferir para la disminución de discapacidad y mortalidad como consecuencia de estas patologías.

Pacheco, Santos Silva, Pinheiro Gordia, Bianchini de Quadros y Petroski, (2014), analizaron la asociación entre el estilo de vida y los determinantes socio demográficos de individuos recién matriculados en una universidad pública del sur de Brasil. Destacan que, la prevalencia del estilo de vida inadecuado fue de 5,3\%. Los resultados en el análisis ajustado, demostraron que los universitarios mayores de 20 años (OR=2,87; IC95 \%:1,37-6,03) y con baja escolaridad materna (de 9 años) (OR=2,23; IC95 \%:1,29-3,88) presentaron un riesgo mayor para adquirir un estilo de vida inadecuado.

Escudero, Muñoz Alferez y Planells del Pozo (2015), analizaron el estilo de vida (consumo de alcohol, tabaco y niveles de actividad física) de mujeres estudiantes de la Universidad de Granada, encontrando que el consumo de alcohol es mayor en el grupo de mayor edad y con preferencia, beben cerveza o vino, sin embargo, el grupo más joven muestra un patrón de consumo centrado en los fines de semana siendo las bebidas destiladas las consumidas preferentemente.

Una tercera parte de la población fuma con un incremento en el número de cigarros conforme aumenta la edad. Existe una correlación positiva entre tabaco y alcohol. El 88.9 \% del grupo de menor edad y el 52.7 \% del grupo de mayor edad tienen una actividad física sedentarialigera. Concluyen en la necesidad de concienciar a la población femenina universitaria sobre los beneficios del abandono del consumo de alcohol y tabaco y la práctica regular de ejercicio físico. Además, sería aconsejable desarrollar protocolos de intervención educativa en el ámbito universitario potenciando los hábitos de vida saludables. 


\section{CONCLUSIÓN}

El recorrido realizado en torno a la temática, que recoge los principales hallazgos de diversas experiencias investigativas, realizadas en los últimos diez años sobre los estilos de vida de población universitaria, permite deducir que, los adultos jóvenes parecen encaminados hacia la adopción de conductas de riesgo. Lo preocupante en este grupo de personas es que, poseen los conocimientos necesarios para llevar a cabo una adecuada conducta pero que, aparentemente, no perciben los riesgos.

La mayor parte de la evidencia es descriptiva, siendo las variables más estudiadas, el consumo de alcohol, consumo de tabaco, hábitos alimenticios, actividad física, hábitos de descanso. No se puede olvidar que, el consumo de drogas no legalizadas, la conducta sexual, accidentes y conductas de prevención de los mismos, deben de ser incorporadas en futuras investigaciones.

Se hace resaltar, que la prevalencia de diabetes mellitus detectada en la ciudad de Managua, es más elevada que la reportada en otros países de Latinoamérica. La prevalencia de hipertensión es comparable a la reportada en otros estudios Latinoamericanos. La situación antes descrita, hace prever que en el futuro se producirá un mayor incremento en la prevalencia de diabetes, a medida que la población envejezca, a menos que se introduzcan estrategias preventivas que faciliten la adopción de estilos de vida saludable.

\section{REFERENCIAS BIBLIOGRÁFICAS}

Aracely, Á. G., Hernández Pozo, M. R., Jimenez Martínez, M. \& Durán Díaz, Á. (2014). Estilos de Vida y Presencia de Síndrome Metabólico en estudiantes Universitarios. Diferecias por sexo. Revista de Psicología.

Cala, M. L. (2010). Estilos de Vida y Salud en Estudiantes de una Facultad de Psicología. Psicología desde el Caribe, 155-178.

Pensamiento Psicológico. (2010). Calidad de vida y Estilos de Vida Saludable en un grupo de estudiantes de pregrado de la ciudad de Lima. 17-38.

Castaño Castrillon, J. J. (2010). Estilos de vida y salud en estudiantes de una facultas de psicología. Psicología del Caribe. 155-178.
Costa, M. \& López , E. (1996). Estilos de Vida y Promoción de la Salud. Madrid: Pirámide.

Dayana, G. L., García Salamanca, G. P., Tapiero Paipa, Y. T. \& Ramos C, D. M. (2012). Determinates de los Estilos de Vida y su Implicación en la Salud de Jóvenes universitarios. Hacia la Promoción de la Salud, 169-185.

Escudero, A. G., Muñoz Alferez, A. \& Planells del Pozo, M. E. (2015). La etapa universitaria no favorece el estilo de vida saludable en las estudiantes granadinas. Nutricion Hospitalaria, 174-179.

Escudero, A. G., Muñoz Alférez, M. J., Planells del Pozo, E. M. \& López Aliaga, I. (2015). 
La etapa Universitaria no favorece el estilo de vida saludable en las estudiantes granadinas . Nutrición Hospitalaria, 975979.

Estilos de Vida de Estudiantes Universitarios admitidos al Pregrado de la Carrera de medicina. (2013). Revista Facultad de Medicina. 51-56.

Fabiola Bacerra Bulla, G. P. (2011). Estilo de vida de estudiantes universitarios adminitidos al pregrado de la carrera de medicina. Revista de la facultad de medicina. 51- 56.

Fundación BBVA. (2007). Libro de la Salud del Hospital Clinic de Barcelona y de la Fundación BBVA. España: Editoreal Nerea SA.

Fundaciòn BBVA. (2007). Libro de la salud del Hospital Clinic de Barcelona y de la Fundacion BBVA. España: Narea SA.

García, V. E., Salazar Arango, P. A., Docal Millán, M. d., Aya Gómez, V. L., Ardila Chacón, M. M. \& Reynaldo, R. (2013). Estilo de vida de los jóvenes y su necesidad de educacion sexual. Colombia: IEMP.

Grimaldo, M. P. (2010). Calidad de vida y estilo de vida saludable en un grupo de estudio de posgrado de la ciudad de Lima . Pensamiento Psicologico. 17-38.

J, R., Pique, J. M. \& Trilla, A. (2007). Libro de la Salud del Hospital Clinico de Barcelona y de la Fundacion BBVA. Bilboa: BBVA.

Laguando, E. \& Gómez Díaz, M. P. (2014). Estilos de Vida Saludable en Estudiantes de Enfermería en la Universidaf Cooperativa de Colombia. Revista Hacia la Promoción de la Salud. 68-83.

Laguna, D. G., García Salamanca, G. P. \& Tapiero Paipa, Y. T. (2012). Detrminanates de los Estilos de Vida y su implicaciòn en la Salud de jóvenes Universitarios. Hacia la Promoción de la Salud. 169-185.

OMS. (2014). ENT Perfil de Países.

Organización Mundial de la Salud. (2014). ENT Perfiles de países. Nicaragua.

Organización Mundial de la Salud. (2014). Informe sobre la situación Mundial de la las Enfermedades no Transmisibles. Ginebra: WHO.

Organización Mundial de la Salud. (2014). Informe Sobre la Situación Mundial de las enfermedades no Transmisibles. OMS. Ginebra: WHO/.

Organización Mundial de la Salud. (2014). Organización Mundial de la Salud - ENT Perfiles de países. Nicaragua.

Pacheco, R. L., Santos Silva, D. A., Pinheiro Gordia, A., Bianchini de Quadros, T. M. \& Petroski, E. L. (2014). Sociodemographic Determinants of University Students Lifestyles. Revista de Salud Pública. 382393.

Pan American Health Organization. (2010). Encuesta de Diabetes, Hipertensión y Factores de Riesgo de Enfermedades Crónicas. Iniciativa Centroamericana de Diabetes (CAMDI). Managua, Nicaragua: Biblioteca Sede OPS - Catalogación en la fuente. 
Ramos, P. (03 de 03 de 2011). grupo. Veny, M. B. (2000). Estilos de Vida y Salud en us.es. Obtenido de: alojamientosv. estudiantes universitarios: La Universidad us.es:s/estudiohbsc/images/pdf/ informaciontema2.pdf como entorno Promotor de la salud. España: UNICS.

T, S. \& Arrivillaga Quintero, I. C. (2004). El Consumo de Alcohol y otras drogas, como parte de los estilos de vida de jóvenes Universitarios. Revista Colombiana de Psicología. 74-89.

Veny, M. B. (2012). Estilos de Vida en Estudiantes Universitarios. Dialnet. 3435. 